\title{
Cutaneous and systemic plasmacytosis on the face: Effective treatment of a case using thalidomide
}

\author{
SHENG FANG, KUI SHAN and AI-JUN CHEN
}

Department of Dermatology, The First Affiliated Hospital of Chongqing Medical University, Chongqing 400016, P.R. China

Received September 9, 2014; Accepted May 27, 2015

DOI: $10.3892 / \mathrm{ol} .2016 .4140$

\begin{abstract}
Cutaneous and systemic plasmacytosis is an exceedingly rare condition that is identified in Japanese individuals in particular. The present study describes the case of a patient of mainland Chinese origin who manifested with red-brown macules, papules and plaques limited to the face. Identifying a therapy for cutaneous and systemic plasmacytosis is quite difficult, however, the present patient showed a good response to low-dose thalidomide. The exact mechanism of action is not yet clear, however, we hypothesize that thalidomide may function through decreasing the secretion of interleukin- 6 and affecting the growth of plasma cells.
\end{abstract}

\section{Introduction}

Cutaneous and systemic plasmacytosis is a rare condition characterized by an infiltration of mature plasma cells in various organ systems, which manifests clinically in the form of red-brown macules, papules and plaques. Since plasmacytosis regularly occurs at extracutaneous sites, the disorder is thus currently known as 'cutaneous and systemic plasmacytosis' (1). This disorder is usually accompanied by fever, anemia, polyclonal hypergammaglobulinemia and superficial lymphadenopathy (2). Cutaneous and systemic plasmacytosis is an uncommon disorder with $<100$ patients described in the literature thus far, the majority of whom are Japanese (3). A previous study suggested that cutaneous and systemic plasmacytosis has a male to female ratio of 1.0:0.6, and an age of onset between 20 and 62 years with a mean and median age of 37 years (4). The condition is usually diagnosed via biopsy; histologically, superficial and deep perivascular and perineural dermatitis with prominent plasma cells is observed (5). Overall, the prognosis of the disorder is favorable, although rare cases have been reported

Correspondence to: Dr Sheng Fang, Department of Dermatology, The First Affiliated Hospital of Chongqing Medical University, 1 Youyi Road, Chongqing 400016, P.R. China

E-mail: fangshengderm@163.com

Key words: hypergammaglobulinemia, lymphadenopathy, plasmacytosis with a more aggressive clinical course, including the development of lymphoma (5). Treatment with immunosuppressive agents of variable potency has been described with a degree of success; however, there is not a common effective treatment (2). The present study reports a case of cutaneous and systemic plasmacytosis on the face that occurred in a patient of Chinese origin. Written informed consent was obtained from the patient.

\section{Case report}

A 50-year-old female from mainland China presented to The First Affiliated Hospital of Chongqing Medical University (Chongqing, China) for evaluation of diffuse, asymptomatic purplish-red macules that had been present on the face for one year. The lesions initially started on the upper lip, and then disseminated to the other areas of the face, nose and forehead, with main dispersal on the lips and perioral area. The patient had previously been diagnosed with seborrheic dermatitis and treated with antihistamine drugs, without any improvement.

A physical examination revealed multiple red-brownish macules, plaques and nodules, measuring 1-2 cm in diameter, distributed symmetrically on the face, particularly the lips and perioral area where the papules had fused to form plaques (Fig. 1). In addition, non-tender peripheral lymphadenopathy was detected in the cervical, axillary and inguinal regions. No hepatosplenomegaly was revealed. There was no family history of plasmacytosis and the patient's general condition was good. Laboratory examinations revealed no abnormalities in complete blood count, serum human immunodeficiency virus antibody level, rapid plasma reagin, antinuclear antibody and extractable nuclear antigen. Urinalysis repeat examinations did not show any evidence of Bence-Jones protein in the urine. A bone X-ray showed no signs of myeloma and a bone marrow smear showed no signs of malignant plasmacytoma. The erythrocyte sedimentation rate was elevated at $105 \mathrm{~mm} / \mathrm{h}$ (normal range, $0-15 \mathrm{~mm} / \mathrm{h}$ ). Serum protein electrophoresis demonstrated polyclonal hypergammaglobulinaemia as follows: 2,120 mg/dl immunoglobulin (Ig)G (normal range, 751-1,560 mg/dl), $633 \mathrm{mg} / \mathrm{dl}$ IgA (range, 82-453 mg/dl) and $311 \mathrm{mg} / \mathrm{dl} \mathrm{IgM}$ (range, 46-304 mg/dl). A skin biopsy taken from the upper lip and stained with haematoxylin and eosin showed a dermal periadnexal diffuse infiltrate, consisting mostly of plasma cells admixed with a few lymphocytes and histiocytes (Fig. 2). 


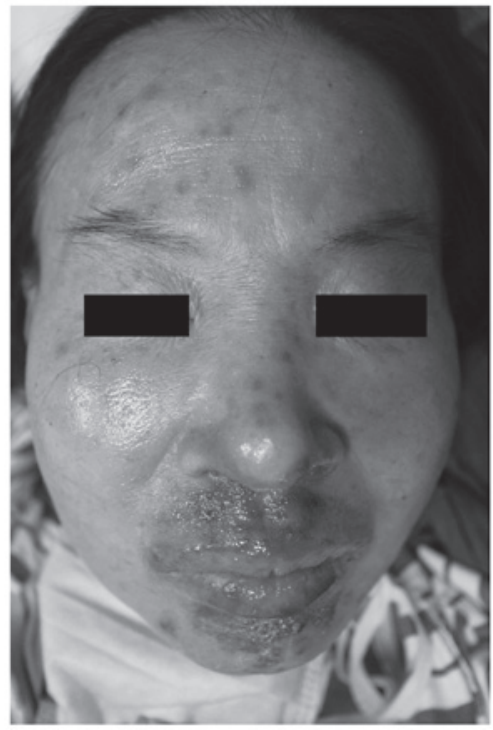

Figure 1. Multiple red-brownish macules present diffusely on the face, particularly on the lips and perioral area.
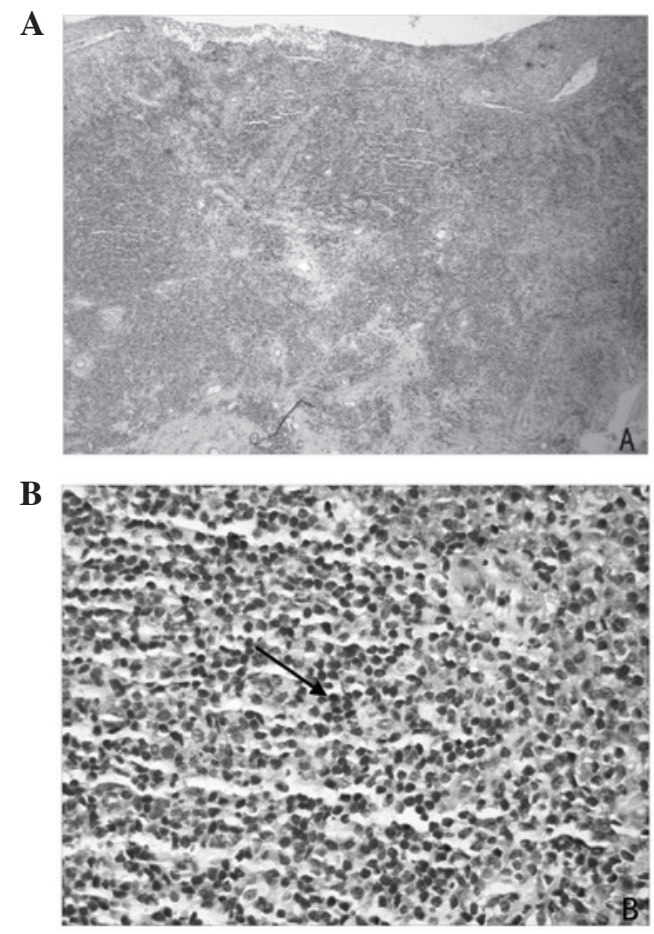

Figure 2. Upper lip skin biopsy. (A) Low-power view revealing dermal diffuse infiltrates (hematoxylin and eosin stain; magnification, x40). (B) High-power view revealing features consisting mostly of plasma cells (shown by the black arrow) admixed with a few lymphocytes and histiocytes (hematoxylin and eosin stain; magnification, $\mathrm{x} 400$ ).

According to the typical clinical features, histopathology results and laboratory examination, a diagnosis of cutaneous and systemic plasmacytosis was made. The patient was prescribed oral thalidomide (Changzhou Pharmaceutical Factory, Changzhou, China) at a dose of $25 \mathrm{mg}$ three times per day $(75 \mathrm{mg} /$ day). One and a half months after the start of the therapy, the skin lesions had improved as the cutaneous plaques had decreased in size. Three months after the start of the therapy, the skin lesions had mostly cleared (Fig. 3).

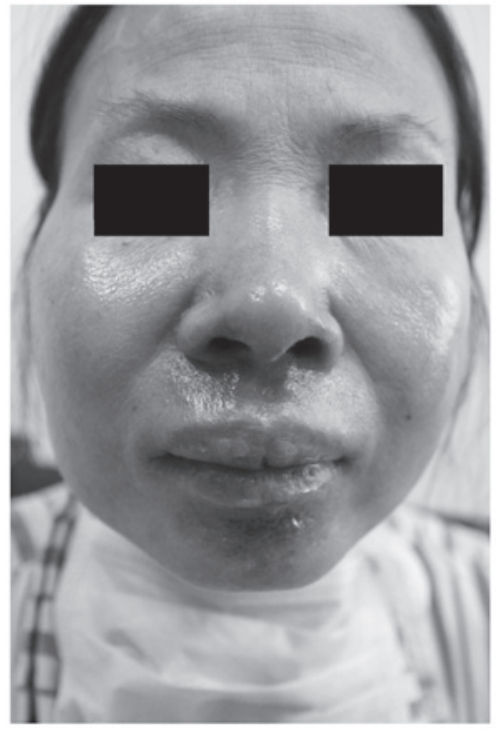

Figure 3. Patient four months after effective treatment with thalidomide.

The patient did not experience any adverse effects. Baseline sensory nerve conduction studies were performed during the therapy as part of the monitoring process for thalidomide-induced peripheral neuropathy. In a recent telephone follow-up, the patient confirmed that the condition continues to improve.

\section{Discussion}

Cutaneous and systemic plasmacytosis was first reported by Yashiro (6) in a study titled 'A kind of plasmocytosis: Primary cutaneous plasmacytoma?' in 1976 and its description was further refined in the 1980s by Kitamura et al (7). To date, the majority of cutaneous and systemic plasmacytosis cases have been observed in Asian populations, and more specifically in Japanese populations; all 41 patients reported by Uhara et al (8) were Japanese. In addition, isolated cases have been reported in Chinese, Korean and Thai populations $(9,10)$. Worldwide, cutaneous and systemic plasmacytosis has only been reported in $\sim 10$ Caucasian patients (11). The typical skin lesions are asymptomatic, brownish, irregularly-shaped macules or plaques, which are mostly disseminated on the trunk. The lesions also can additionally involve the face or limbs. However, the presentation of the current patient was slightly different in that the lesions were just limited to the face. Aside from the compulsory cutaneous manifestation, polyclonal hypergammaglobulinemia and lymphadenopathy are the most frequent symptoms of the disease (9). The majority of patients are asymptomatic, although individuals with systemic involvement may present with constitutional symptoms, including weight loss and fatigue (3).

Given the rarity of cutaneous and systemic plasmacytosis, no controlled therapeutic trials have thus far been conducted, and a commonly effective treatment has not been reported. Generally, the therapy for cutaneous and systemic plasmacytosis has been quite disappointing until now. Studies have shown that the most commonly used topical or intralesional 
therapies performed with corticosteroids result in no, slight or an only transient improvement $(12,13)$. Tacrolimus and pimecrolimus have been applied as treatment with different effects. Ma et al (10) and Miura et al (14) reported that there was no or only slight benefit when treated with tacrolimus, whereas Hafner et al (15) recorded effective treatment with pimecrolimus in one case. Other available topical treatments for cutaneous plasmacytosis include radiotherapy, photodynamic therapy and psoralen combined with ultraviolet A (16). In individual cases, patient have been treated with cyclophosphamide, vincristine, prednisone and rituximab chemotherapy with only partial, transient resolution of the lesions (17). Lee et al (18) showed that systemic plasmacytosis was improved with melphalan treatment.

To the best of our knowledge, the present study reports the first case of an effective treatment of cutaneous and systemic plasmacytosis with thalidomide. The main adverse effects of thalidomide are sedation, fatigue, constipation, peripheral neuropathy and thromboembolic phenomena; however, thalidomide treatment was well tolerated in the present patient (19).

Cutaneous and systemic plasmacytosis is rare with an unknown etiology. Multiple theories have been proposed, including the elevation of interleukin (IL)-6 (13). The variability of humans geographically or IL-6 genetic polymorphisms may possibly explain the frequency of cutaneous and systemic plasmacytosis in individual regions. Furthermore, Haque et al (20) hypothesized that due to the local production of IL-6, long-lived plasma cells originate and survive in the environment of the skin, and that such patients may therefore respond to agents able to interfere with the activity of IL-6. Thalidomide stimulates the cytotoxic functions of $\mathrm{T}$ lymphocytes, thus limiting the immunosuppressive function of regulatory $\mathrm{T}$ cells and significantly altering the immunological profile by inhibiting the release of TNFa and IL-6 (21). We hypothesized that through decreased secretion of IL-6, thalidomide may affect the growth of plasma cells, which could be beneficial for the treatment of cutaneous and systemic plasmacytosis.

Overall, the present study showed that systemic thalidomide was effective in the treatment of cutaneous and systemic plasmacytosis. The exact mechanism of action is unknown, but the drug may act directly on plasma cells or alter the immunological profile. However, further studies, including randomized controlled trials, are required to confirm the benefit of this association. Although the present study achieved great improvements with low-dose thalidomide at $75 \mathrm{mg}$ /day, individual dosages may be different. The addition of other agents, such as dexamethasone, may also improve the response rates. With monitoring of nerve conduction studies, we believe that thalidomide and topical tacrolimus could be considered as a safe therapeutic option for patients with cutaneous and systemic plasmacytosis.

\section{References}

1. Tada Y, Komine M, Suzuki S, Kikuchi K, Sasaki M, Kaneko N and Tamaki K: Plasmacytosis: Systemic or cutaneous, are they distinct? Acta Derm Venereol 80: 233-235, 2000.

2. Carey WP, Rico MJ, Nierodzik M and Sidhu G: Systemic plasmacytosis with cutaneous manifestations in a white man: Successful therapy with cyclophosphamide/prednisone. J Am Acad Dermatol 38: 629-631, 1998.

3. Honda R, Cerroni L, Tanikawa A, Ebihara T, Amagai M and Ishiko A: Cutaneous plasmacytosis: Report of 6 cases with or without systemic involvement. J Am Acad Dermatol 68: 978-985, 2013.

4. Leonard AL, Meehan SA, Ramsey D, Brown L and Sen F: Cutaneous and systemic plasmacytosis. J Am Acad Dermatol 56 (Suppl): S38-S40, 2007.

5. Jayaraman AG, Cesca C and Kohler S: Cutaneous plasmacytosis: A report of five cases with immunohistochemical evaluation for HHV-8 expression. Am J Dermatopathol 28: 93-98, 2006.

6. Yashiro A: A kind of plasmacytosis: Primary cutaneous plasmacytoma? Jpn J Dermatol 86: 910, 1976.

7. Kitamura K, Tamura N, Hatano H, Toyama K, Mikata A and Watanabe S: A case of plasmacytosis with multiple peculiar eruptions. J Dermatol 7: 341-349, 1980.

8. Uhara H, Saida T, Ikegawa S, Yamazaki Y, Mikoshiba H, Nijoh S, Kitano K and Koh CS: Primary cutaneous plasmacytosis: Report of three cases and review of the literature. Dermatology 189: 251-255, 1994

9. Wagner G, Rose C, Klapper W and Sachse MM: Cutaneous and systemic plasmocytosis. J Dtsch Dermatol Ges 11: 1161-1167, 2013.

10. Ma HJ, Liu W, Li Y, Zhao G, Meng RS and Li DG: Cutaneous and systemic plasmacytosis: A Chinese case. J Dermatol 35: 536-540, 2008

11. González-López MA, González-Vela MC, Blanco R, Fernández-Llaca $\mathrm{H}$ and Val-Bernal JF: Cutaneous plasmacytosis limited to the extremities in a white patient: An unusual clinical picture. Cutis 86: 143-147, 2010.

12. Ahn JJ, Yang YS, Shin MK, Lee SW and Kim NI: Case of isolated benign primary cutaneous plasmacytosis in a child. J Dermatol 38: 364-367, 2011.

13. Yamamoto T, Katayama I and Nishioka K: Increased plasma interleukin-6 in cutaneous plasmacytoma: The effect of intralesional steroid therapy. Br J Dermatol 137: 631-636, 1997.

14. Miura H, Itami S and Yoshikawa K: Treatment of facial lesion of cutaneous plasmacytosis with tacrolimus ointment. J Am Acad Dermatol 49: 1195-1196, 2003.

15. Hafner C, Hohenleutner U, Babilas P, Landthaler M and Vogt T: Targeting T cells to hit B cells: Successful treatment of cutaneous plasmacytosis with topical pimecrolimus. Dermatology 213: 163-165, 2006.

16. Tzung TY, Wu KH, Wu JC and Tseng HH: Primary cutaneous plasmacytosis successfully treated with topical photodynamic therapy. Acta Derm Venereol 85: 542-543, 2005.

17. Amin HM, McLaughlin P, Rutherford CJ, Abruzzo LV and Jones D: Cutaneous and systemic plasmacytosis in a patient of Asian descent living in the United States. Am J Dermatopathol 24: 241-245, 2002.

18. Lee DW, Choi SW, Park JW and Cho BK: Systemic plasmacytosis: A case which improved with melphalan. J Dermatol 22: 205-209, 1995.

19. Berrebi A, Feldberg E, Spivak I and Shvidel L: Mini-dose of thalidomide for treatment of primary myelofibrosis. Report of a case with complete reversal of bone marrow fibrosis and splenomegaly. Haematologica: 92: e15-e16, 2007.

20. Haque M, Hou JS, Hisamichi K, Tamada K, Cusack CA, Abdelmalek M, Brown RE and Vonderheid EC: Cutaneous and systemic plasmacytosis vs. cutaneous plasmacytic castleman disease: Review and speculations about pathogenesis. Clin Lymphoma Myeloma Leuk 11: 453-4561, 2011.

21. Semeraro M, Vacchelli E, Eggermont A, Galon J, Zitvogel L, Kroemer G and Galluzzi L: Trial Watch: Lenalidomide-based immunochemotherapy. Oncoimmunology 2: e26494, 2013. 